\title{
An Unusual Presentation of a Low Grade Follicular Lymphoma Masquerading as a Meningioma
}

\author{
MacCann R*, Gleeson J P, Aird JJ , Beausang A, \\ Breathnach O, Morris PG and Grogan W \\ Department of Medical Oncology, Beaumont Hospital, \\ Dublin, Ireland \\ *Corresponding author: Rachel MacCann, \\ Department of Medical Oncology, Beaumont Hospital, \\ Dublin, Ireland
}

Received: J une 28, 2018; Accepted: July 30, 2018; Published: August 06, 2018

\begin{abstract}
The central nervous system (CNS) is an important area of involvement for both high-grade, aggressive primary and secondary lymphomas. Follicular lymphoma typically represents a low-grade histology. Here, we describe a case of higher-grade follicular lymphoma with CNS involvement, where the complicated and unsuspected diagnosis was prompted following a single seizure event.

Keywords: Indolent lymphoma; Follicular Iymphoma; Diffuse large B-cell lymphoma; Secondary CNS involvement; Parenchymal CNS involvement
\end{abstract}

\section{Introduction}

The CNS is often involved with both primary and secondary lymphomas, but different types of lymphoma manifest considerably different patterns of disease. The differential diagnosis of patients presenting with a CNS form of non-Hodgkin Lymphoma can thus be broad and variable. Typically, patients present with progressive encephalopathy or focal neurologic deficits accompanied by enhancing abnormalities on brain imaging. In light of this, we report a rare case of follicular lymphoma with diffuse lymphadenopathy following a single seizure episode.

\section{Case Presentation}

$\mathrm{RM}$ is a 41 year-old lady who presented to a peripheral hospital in September 2016 following a seizure episode. She had no history of previous seizures, no headaches, no neurological deficits and past medical history was non-contributory. She took no regular medications. She was an ex-smoker with a 30 pack-year history, had no history of illicit drug use and was a non-drinker. She worked parttime and lives with her partner and 13 year-old son. She had a strong neuro-oncological family history, with a maternal aunt and paternal uncle who had unspecified brain tumours and a paternal aunt had a meningioma. Her brother had acute myeloid leukaemia (AML). On examination, Glasgow Coma Scale (GCS) score was 15/15 with no focal neurological signs, no cerebellar signs, and no palpable lymphadenopathy. Cardiovascular and respiratory examinations were normal. A full blood count and metabolic profile was within the normal range except for mild anaemia. Lactate dehydrogenase (LDH) was normal.

A magnetic resonance imaging (MRI) scan, found a right parietal extra-axial mass which appeared to arise from the dural and so she was transferred to a tertiary neurosurgical centre, for further work up and surgery, with the initial working diagnosis of a seizure-inducing meningioma. RM underwent a parietoccipital craniotomy with resection of the right parietal lesion. She recovered well following surgery, with no complications or neurological deficits.

Formalin fixed paraffin embedded sections from the dural lesion demonstrated a cellular proliferation with follicular and diffuse

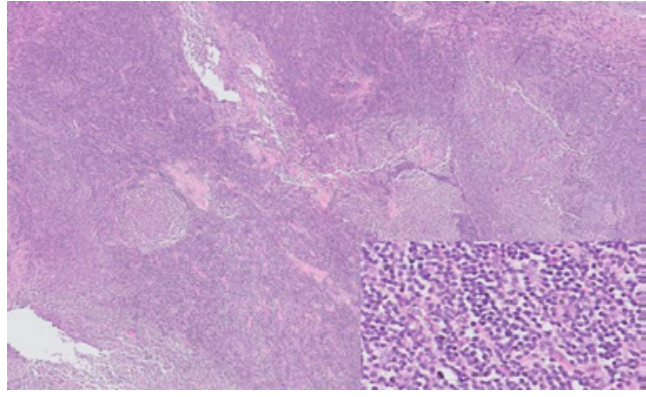

Figure 1: Low power view of a H\&E stained section from the dura showing a cellular lesion with a follicular and diffuse architecture. Inset: the cells are small, monotonous with irregular nuclei and scant cytoplasm.

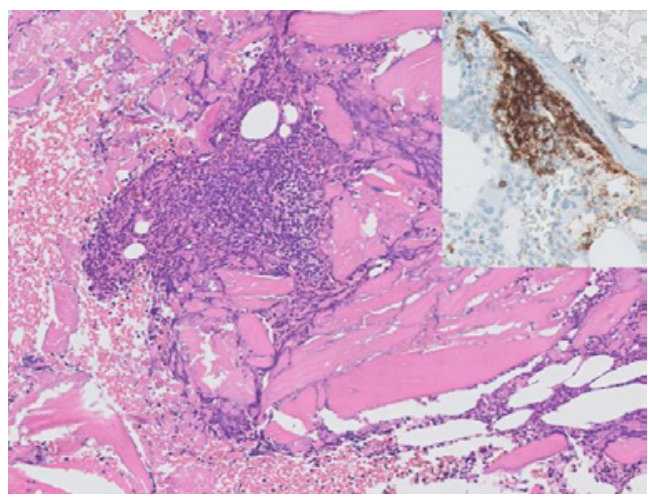

Figure 2: $\mathrm{H}$ and $\mathrm{E}$ stained section from bone marrow trephine biopsy showing a single lymphoid aggregate. Inset: CD20 immunohistochemistry demonstrating positive staining within the lymphoid aggregate.

architecture (Figure 1). The cells were small in size with irregular nuclei, condensed chromatin and scant cytoplasm. There were less than 15 centroblasts per high power field (hpf). Mitoses were not readily identified. The neoplastic cells were positive for $\mathrm{CD} 20, \mathrm{CD} 10$, BCL2, BCL6 and CD23 and negative for CD5, cyclin D1 and CD138. CD21 highlighted expanded and distorted follicular dendritic cell mesh works. CD3 and CD5 stained scattered background reactive T lymphocytes. MIB-1 proliferation index was approximately $40 \%$. No 


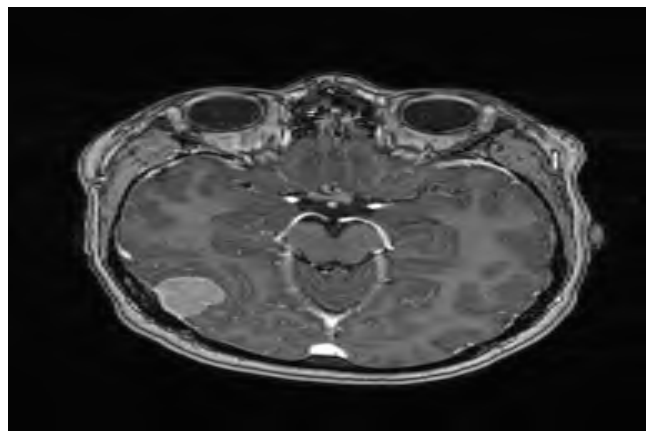

Figure 3: Axial T1 weighted post-contrast MRI image showing what appears to be a homogenous dural-based lesion causing compression of the right temporal lobe.

evidence of a higher grade of lymphoma was identified. Fluorescent in-situ hybridisation analysis showed the presence of a $\mathrm{t}(14 ; 18)$ (q32;q21) translocation. This was felt to be a somewhat inconclusive immunoprofile, however upon discussion at the Lymphoma Multidisciplinary Meeting (MDM), a diagnosis of follicular lymphoma was made. In the clinical context, these morphological, immunohistochemical and molecular features were diagnostic of a follicular lymphoma, grade 1-2, follicular and diffuse subtype. Surprisingly, further examination also showed indolent invasion of the cerebral cortex.

In light of this pathological diagnosis, a complete staging work up was conducted. Computed tomography (CT) of the thorax, abdomen and pelvic areas showed enlarged mesenteric, left axillary and mediastinal lymph nodes with a large nodal mass in the pericoeliac, para-aortic areas and a small pelvic side wall lymph node. A positron emission tomography (PET) scan confirmed widespread lymphadenopathy above and below the diaphragm.A bone marrow trephine biopsy showed a single aggregate of $\mathrm{CD} 20$ positive lymphocytes within the bone marrow suspicious for involvement by follicular lymphoma (Figure 2), confirming Stage IV disease.

Given the cerebral cortex invasion and her presentation with a seizure, localized treatment of the involved cerebral area with stereotactic radiation was planned in the first instance. She underwent a treatment course of 25 Gy 5 Fr of Stereotactic Radiosurgery (SRS) to the right posterior cerebellum.

She subsequently underwent 6 cycles of Rituximab, Cyclophosphamide, Doxorubicvin, Vincristine and Prednisolone (R-CHOP) chemotherapy every 21 days with high dose methotrexate on Day 10 of each cycle. She tolerated the treatment course well, experiencing some side effects of fatigue, peripheral neuropathy, sweating and bleeding gums, but missed every second Methotrexate cycle due to neutropaenia, and these were added on at the end.

A CT of the thorax, abdomen and pelvis was conducted two months into treatment and found resolution of bilateral axillary lymph nodes, resolution of subpectoral lymph nodes and sub-diaphragmatic and left internal mammary lymph nodes were no longer seen. MRI upon treatment completion in April 2017 found no residual abnormal signal at the right parieto-occipital resection site. Furthermore, a CT of the thorax, abdomen and pelvis found complete resolution of previously described lymph nodes. Maintenance treatment with

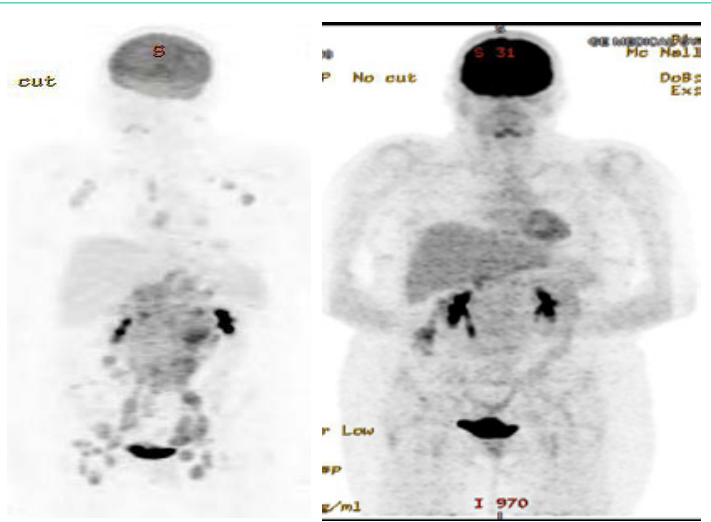

Figure 4: Pre (left), and Post (right) treatment PET scans showing resolution of bilateral axillary lymph nodes, subpectoral lymph nodes, sub-diaphragmatic and left internal mammary lymph nodes.

Rituximab every 2 months for 2 years was commenced with a follow up PET scan that showed a complete remission (CR).

\section{Discussion}

Here we describe the unusual case of a patient with an indolent variant of follicular lymphoma, grade 1-2 with surprisingly aggressive features, including invasion of the cerebral cortex. As a result of diagnostic confusion regarding the etiology, the mass was surgically excised rather than biopsied, as is standard for any suspected CNS lymphoma. However, because the lesion was fully excised, the invasion of the cerebral cortex was identified and this may have been missed had a stereotactic biopsy only been performed. This demonstrated a follicular lymphoma with retention of Bcl-2 and Bcl6 immunoreactivity.

Follicular lymphoma is a subtype of Non-Hodgkin Lymphoma (NHL). It is largely an indolent B cell lymphoproliferative disorder of transformed follicular centre B cells. It is the most common subtype of indolent NHL and comprises approximately $22 \%$ of all cases [1]. Follicular lymphoma is characterized by diffuse lymphadenopathy, marrow involvement, splenomegaly, and less commonly extra nodal sites. CNS involvement by NHL occurs in approximately $8 \%$ of the cases, and can present a diagnostic challenge [1]. The clinical presentation and neuroimaging characteristics may mimic those of other progressive neurologic disorders, including primary brain tumor, demyelinating disease, autoimmune or paraneoplastic syndromes, or CNS infection [1]. For indolent follicular lymphoma, the literature on CNS involvement is scarce. Descriptions to date are limited to isolated case reports, with a varying clinical course for each case [4-7].

Systemic indolent lymphomas are the least frequent type of systemic lymphoma to involve the CNS. CNS invasion occurs in 3\% of these patients, mainly after histological transformation to highgrade lymphoma. Patients typically have a poor prognosis. Predictive factors for prognosis are aggressive histology, such as Burkitt's and lymphoblastic lymphomas; involvement of the paranasal sinuses and testicles; increased LDH, bone marrow involvement, and multiple extra nodal site involvement [8]. MIB-1 immunohistochemistry can help to stratify B-cell NHL and showed a significant increase in proliferation index (PI) with tumor aggressiveness [9]. In light of this, 
CNS prophylaxis is recommended in patients with high grade NHL or for patients with markers of higher risk [10].

Although treatment for Follicular Lymphoma is typically determined based on the extent of disease and staging, the rarity of these cases presents a clinical challenge. Chemotherapy, radiation, surgery, or a combination of these modalities has been used as part of its treatment $[11,12]$. The R-CHOP chemotherapy regimen is by far the most common, showing longer overall survival rates when compared to other regimens [13]. Follicular lymphoma characteristically responds well to first-line chemotherapy but typically manifests repeated relapses with the need for recurrent therapeutic interventions, with disease-free intervals becoming progressively shorter. The addition of Rituximab in first-line treatment for follicular lymphoma has been shown to significantly to improve progression-free survival [14].

\section{Conclusion}

Parenchymal brain involvement is an atypical pattern of spread for systemic lymphomas. More notably, follicular lymphomas are one of the least frequent types of indolent lymphomas to develop clinically apparent, secondary CNS spread. The presentation of an indolent follicular lymphoma with invasion into the brain parenchyma within the brain parenchyma is rare. Its presentation as a single seizure is unique and prompted diagnostic perplexity. Following surgical resection, our patient underwent stereotactic radiation to the resection site, then completed her R-CHOP regimen with high dose methotrexate and has commenced two years of consolidation rituximab therapy. While treatment should obviously be tailored for individual cases, we suggest this as an appropriate regimen in relevant cases.

\section{References}

1. Armitage JO, Weisenburger DD. New approach to classifying non-Hodgkin's lymphomas: clinical features of the major histologic subtypes. Non-Hodgkin's Lymphoma Classification Project. J Clin Oncol. 1998; 16: 2780-2795.

2. Zelenetz AD, Abramson JS, Advani RH, Andreadis CB, Byrd JC, Czuczman MS, et al. NCCN Clinical Practice Guidelines in Oncology: non-Hodgkin's lymphomas. J Natl Compr Canc Netw. 2010; 8: 288-334.
3. Hunt MA, Jahnke K, Murillo TP, Neuwelt EA. Distinguishing primary central nervous system lymphoma from other central nervous system diseases: a neurosurgical perspective on diagnostic dilemmas and approaches. Neurosurg Focus. 2006; 21: E3.

4. Grupka NL, Seinfeld J, Ryder J, Lillehei KO, Kleinschmidt-Demasters BK. Secondary central nervous system involvement by follicular lymphoma: case report and review of the literature. Surg Neurol. 2006; 65: 590-594.

5. Liang RHS, Woo EKW, Yu YL, Todd D, Chan TK, Ho FC, et al. Central nervous system involvement in non-Hodgkin's lymphoma. Eur J Cancer Clin Oncol. 1989; 25: 703-710.

6. Recht L, Straus DJ, Cirrincione C, Thaler H, Posner JB. Central nervous system metastases from non-Hodgkin's lymphoma: treatment and prophylaxis. Am J Med. 1988; 84: 425-435

7. Liang RHS, Chiu E, Loke SL. Secondary central nervous system involvement by non-Hodgkin's lymphoma: the risk factors. Hematol Oncol. 1990; 8: 141145.

8. Hollender A, Kvaloy S, Nome O, Skovlund E, Lote K, Holte H. Central nervous system involvement following diagnosis of non-Hodgkin's lymphoma: a risk model. Ann Oncol. 2002; 13: 1099-1107.

9. Spectre G, Gural A, Amir G, Lossos A, Siegal T, Paltiel O. Central nervous system involvement in indolent lymphomas. Ann Oncol. 2005; 16: 450-454.

10. Karadurmus, N, Ataergin S, Erdem G, Cakar M, Emer O, Ozaydin S, et al. A Rare Presentation of Follicular Lymphoma: Cerebellar Involvement Successfully Treated with a Combination of Radiotherapy and Chemotherapy. Cancer Res Treat. 2013; 45: 234-238.

11. Frederico M. R-CVP VS R-CHOP VS R-FM for the initial treatment of patients with advanced stage follicular lymphoma - preliminary results of foll05 IIL trial. Poster session presented at: 11th International Conference on Malignant Lymphoma; 2011 June 15-18. Lugano, Switzerland. Ann Oncol. 2011; 2 : 1839.

12. Salles G, Seymour JF, Offner F, López-Guillermo A, Belada D, Xerri L, et al. Rituximab maintenance for 2 years in patients with high tumour burden follicular lymphoma responding to rituximab plus chemotherapy (PRIMA): a phase 3, randomised controlled trial; The Lancet. 2011; 377: 42-51.
Ann Hematol Oncol - Volume 5 Issue 6 - 2018

ISSN : 2375-7965 | www.austinpublishing group.com

MacCann et al. (C) All rights are reserved
Citation: MacCann R, Gleeson JP, Aird JJ, Beausang A, Breathnach O, Morris PG, et al. An Unusual Presentation of a Low Grade Follicular Lymphoma Masquerading as a Meningioma. Ann Hematol Oncol. 2018; 5(6): 1211. 